\title{
DOUBLE NORMALS AND TANGENT NORMALS FOR POLYGONS
}

\author{
BENJAMIN HALPERN ${ }^{1}$
}

\begin{abstract}
Given a polygonal closed plane curve $\gamma$. Each segment of $\gamma$ has a tangent direction and a normal direction; each vertex of $\gamma$ has a cone of tangent directions and a cone of normal directions. Formulas are established connecting the numbers of various kinds of straight lines which either intersect $\gamma$ twice in a normal direction, or once in a normal direction and once in a tangent direction.
\end{abstract}

1. Introduction. In a recent paper, Thomas Banchoff $[1]$ established a polyhedral analog of a theorem of Fabricius-Bjerre [3]. This theorem gives an equation involving the numbers of double tangents, crossings, and inflection points of a closed plane curve. The author rediscovered this theorem and gave a proof of it and of several similar theorems involving double normals and tangent normals to closed plane curves in [2]. The purpose of this note is to show that the other theorems of [2] have polyhedral analogs and that these polyhedral analogs have elementary proofs analogous to Banchoff's proof of the theorem of Fabricius-Bjerre.

Banchoff uses an appealing form of proof in [1]. He first notes that the equation in question holds for a special case. Then he shows that the general case can be deformed into the special case in a very regular or controlled way. Finally, he sees how the quantities in question change as one passes exceptional position in the deformation and notes that the changes cancel out in the equation.

We start with the smooth versions established in [2]. The polyhedral versions are proved by rounding the corners to get a smooth approximation. $\mathrm{Fi}$ nally, we note that since the polyhedral versions are true and regular deformations to special cases exist, proofs of the type described in the preceding paragraph must exist.

Received by the editors June 26, 1973. $57 C 35$.

AMS (MOS) subject classifications (1970). Primary 53C99; Secondary 53A05,

Key words and phrases. Polygonal plane curve, double normal.

1 This research was partially supported by National Science Foundation Grant GP33756. 
2. Definitions. Following Banchoff [1] we define a polygon to be a map ping $X:[a, b] \rightarrow E^{2}$ of a closed interval into the Euclidean plane such that for some finite subdivision, $a=t_{0}<t_{1}<\cdots<t_{m}=b$, the mapping $X$ restricted to the interval $t_{i} \leq t \leq t_{i+1}$ is linear. The points $X\left(t_{i}\right)$ are abbreviated $X_{i}$ and are called the vertices of the polygon. If $X_{0}=X_{m}$, then $X$ is a closed $m$-gon and in this case we consider all induces to be reduced modulo $m$. A polygon $X$ is said to be general if the vertices $\left\{X_{i} \mid i=0,1, \ldots\right.$, $m=1\}$ are in general position, so no three vertices are collinear. We distinguish two new types of general polygons. First, a polygon $X$ is n-general if it is general and no two edges are parallel, and for each $i, j, i \neq j$, segment $\left[X_{i}, X_{j}\right]$ is not perpendicular to the edge $\left[X_{j}, X_{j+1}\right]$. Second, a polygon $X$ is tn-general if it is general and no two edges are perpendicular.

The key to finding polyhedral analogs to the theorems of [2] involving normals is to find the polyhedral analog to normal lines. We are guided by the following idea. Take a vertex of a polyhedral curve and replace a neighborhood of it by an arc of a very small circle as shown in Figure 1. This suggests the following definition.

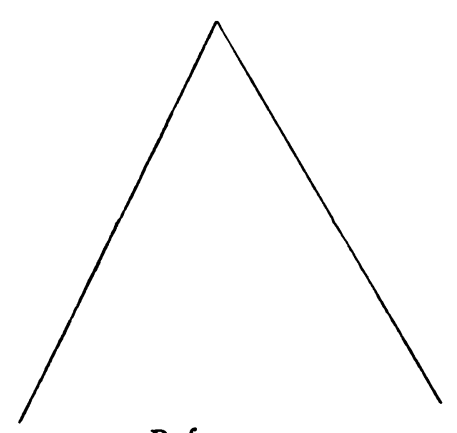

Before

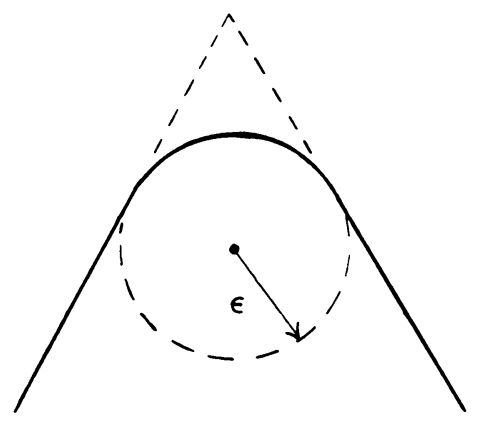

After

\section{Figure 1}

We say that a segment $\left[X_{i}, Q\right]$ is normal to $X$ at the vertex $X_{i}$ provided either

$$
\left[\left(X_{i-1}-X_{i}\right) \cdot\left(Q-X_{i}\right)>0 \text { and }\left(X_{i+1}-X_{i}\right) \cdot\left(Q-X_{i}\right)>0\right]
$$

or

$$
\left[\left(X_{i-1}-X_{i}\right) \cdot\left(Q-X_{i}\right)<0 \text { and }\left(X_{i+1}-X_{i}\right) \cdot\left(Q-X_{i}\right)<0\right] \text {. }
$$

Set

$$
I_{t n}=\left\{(i, j) \mid\left[X_{i}, X_{j}\right] \text { is tangent to } X \text { at } X_{i} \text { and normal to } X \text { at } X_{j}\right\}_{\bullet}
$$


For $(i, j) \in I_{t n}$ define

and

$$
\sigma(i, j)=\left\{\begin{aligned}
1 & \text { if }\left(X_{i}-X_{j}\right) \cdot\left(X_{j+1}-X_{j}\right)>0 \\
-1 & \text { if }\left(X_{i}-X_{j}\right) \cdot\left(X_{j+1}-X_{j}\right)<0
\end{aligned}\right.
$$

Set:

$$
t(i, j)=\left\{\begin{array}{cl}
1 & \text { if }\left(X_{i}-X_{j}, X_{i+1}-X_{i}\right) \text { is a right-handed frame } \\
-1 & \text { if }\left(X_{i}-X_{j}, X_{i+1}-X_{i}\right) \text { is a left-handed frame. }
\end{array}\right.
$$

$$
\begin{gathered}
I_{t n}^{( \pm)}={ }^{\sharp}\left\{(i, j) \in I_{t n} \mid \sigma(i, j) \cdot t(i, j)=\left(\begin{array}{c}
1 \\
-1
\end{array}\right)\right\} ; \\
I I_{t n}^{( \pm)}={ }^{\#}\left\{(i, j) \mid \exists \text { a point } P \text { on }\left[X_{j}, X_{j+1}\right] \ni\left[X_{i}, P\right]\right. \\
\text { is tangent to } X \text { at } X_{i},\left(X_{i}-P\right) \cdot\left(X_{j+1}-X_{j}\right)=0, \\
\text { and } \left.\left(X_{i}-P, X_{i+1}-X_{i}\right) \text { is a right (left-)-handed frame }\right\} ; \\
I_{n}^{( \pm)}={ }^{\#}\left\{(i, j) \mid 0 \leq i<j \leq m-1,\left[X_{i}, X_{j}\right] \text { is normal to } X_{j}\right. \\
\text { at } X_{i} \text { and at } X_{j}, \text { and }\left[\left(X_{i+1}-X_{i}\right) \cdot\left(X_{j}-X_{i}\right)\right] . \\
\left..\left[\left(X_{j+1}-X_{j}\right) \cdot\left(X_{i}-X_{j}\right)\right]>(<) 0\right\} ;
\end{gathered}
$$

$I I_{n}^{( \pm)}={ }^{\sharp}\left\{(i, j) \mid 0 \leq i, j \leq m-1, \exists\right.$ a point $P$ on the edge $\left[X_{j}, X_{j+1}\right] \ni$ $\left[X_{i}, P\right]$ is normal to $X$ at $X_{i}$ and $\left[X_{i}, P\right]$ is perpendicular to the edge $\left[X_{j}, X_{j+1}\right]$, and $\left.\left(X_{i+1}-X_{i}\right) \cdot\left(P-X_{i}\right)<(>) 0\right\}$.

Finally, let $C$ be the number of crossings, i.e. pairs of indices $(i, j)$ with $0 \leq i<j \leq m-1$ such that the edge $\left[X_{i}, X_{i+1}\right]$ meets the edge $\left[X_{j}, X_{j+1}\right]$.

\section{Results.}

Theorem 1. If $X$ is n-general, then $I_{n}^{+}-I_{n}^{-}+I I_{n}^{+}-I I_{n}^{-}+C=0$.

Theorem 2. If $X$ is tn-general, then $I_{t n}^{+}-I_{t n}^{-}+I I_{t n}^{+}-I I_{t n}^{-}=0$.

Proofs of Theorems 1 and 2. The differentiability assumptions in [2] were only needed in neighborhoods of the coordinates of double normal pairs, double tangent pairs, and crossings. At all other points only continuity of the first derivative was used. Replace $X$ by $X_{\epsilon}$ where $X_{\epsilon}$ is obtained from $X$ by rounding the vertices as in Figure 1. Making $\epsilon$ sufficiently small and noting that $X$ is in the appropriate general position, it is easily seen that the corre- 
sponding numbers for $X$ and $X_{\epsilon}$ are the same. Hence Theorems 1 and 2 follow from Theorems 2 and 3 of [2]. Q.E.D.

Theorem 1 of [1] has a similar proof. But here one must also replace the edges by slightly curved lines in addition to rounding the vertices. The generalizations to arbitrary 1-dimension compact closed manifolds carry over in a like manner.

In [1, Lemmas 1, 2], Banchoff establishes the existence of a "nice" piecewise linear deformation from an arbitrary general closed polygon to one of an especially simple form for double tangents. Analogous results for $n$-general and $t n$-general polygons are easily proved by the same techniques as used in [1, Lemmas 1, 2]. Of course, different but analogous concepts of "nice piecewise linear deformation" must be used for $n$-general and $t n$-general polygons. Since Theorems 1 and 2 have now been established we can be sure that as one passes an exceptional position in one of these "nice" deformations, the changes in the relevant quantities cancel in the appropriate equation. Hence there must exist proofs of Theorems 1 and 2 of the same form as Banchoff's proof.

\section{REFERENCES}

1. T. Banchoff, Global geometry of polygons. I: The theorem of Fabricius-Bjerre, Proc. Amer. Math. Soc. 45 (1974), 237-241.

2. B. Halpern, Global theorems for closed plane curves, Bull. Amer. Math. Soc. 76 (1970), 96-100. MR 41 \#7541.

3. Fr. Fabricius-Bjerre, On the double tangents of plane closed curves, Math. Scand. 11 (1962), 113-116. MR 28 \#439.

DEPARTMENT OF MATHEMATICS, INDIANA UNIVERSITY, BLOOMINGTON, INDIANA 47401 